\title{
Outcome assessment of intervention on appropriateness of antibiotic use among geriatric patients: A prospective interventional study from a tertiary care referral hospital
}

\author{
Dilip Chandrasekhar ${ }^{\mathrm{a}, *}$, Harsha Manaparambil ${ }^{\mathrm{a}}$, Jafer Chalil Parambil ${ }^{\mathrm{b}}$ \\ ${ }^{a}$ Department of Pharmacy Practice, Al Shifa College of Pharmacy, Kizhattur, Perinthalmanna, Malappuram, 679325, Kerala, India \\ ${ }^{\mathrm{b}}$ Dept of General Medicine, Kims Alshifa Hospital Pvt Ltd, Perinthalmanna, Malappuram, Kerala, India
}

\section{A R T I C L E I N F O}

\section{Keywords:}

Bacteriological test

Geriatric population

Gyssens criteria

Inappropriateness

Quality

Indicators

\begin{abstract}
A B S T R A C T
Objective: To assess the inappropriate use of antibiotics in geriatric patients and to assess the impact of clinical pharmacist intervention in reducing the inappropriate use of antibiotics.

Methodology: A prospective intervention study of 12 months duration was conducted among 180 geriatric patients who were on treatment with antibiotics for more than 2 days. The outcome measures were compliance with Gyssens criteria and National guidelines for antimicrobial use 1.0(2016). Reason for inappropriateness and quality indicators were also assessed to optimize the antibiotic use and to develop adult traffic light signal for antibiotics.

Results: The mean stay duration was found to be $7.52 \pm 4.47$ days, reduced to $4.93 \pm 1.72$ days in after intervention. Most of indication for antibiotics was respiratory tract infections (30.6\%). In the pre intervention, $70 \%$ antibiotic therapy proved inappropriate which reduced to $48.8 \%$ inappropriateness in the post intervention $(\mathrm{p}=0.004)$. Most common reason given for inappropriateness was wrong drug duration (pre: 41.2\%, post: $27.8 \%)$. There was a poor compliance with the national guidelines for antibiotic use in the pre intervention (15\%) which increased to $25.6 \%$ in post intervention. Most of the therapy was given prophylactically and only $30.55 \%$ ordered bacteriological test before starting treatment.

Conclusion: The study concluded that prescribing patterns do not comply with the recommended standards of care. Since the intervention has a positive impact on inappropriateness the hospital should develop a system to monitor and regulate the use of antibiotics in geriatrics to promote the rational use of antibiotics.
\end{abstract}

\section{Introduction}

Antibiotics represent a major class of antimicrobial agents use widely in infectious diseases which is a major cause of morbidity and mortality in developing countries. Antibiotics account for $15-30 \%$ of drug expenditures, the largest of any therapeutic group of drugs. The increased use of antimicrobial drugs has coincided with the emergence of antimicrobial resistance, which constitutes an important clinical, economic and public health problem. Resistant pathogens increase healthcare associated expense, complicate therapy, and make treatment failure more likely. Therefore there has been a growing attentiveness to rational use of antimicrobials since the 1990s.

Special consideration of antibiotic prescription should be taken in geriatric population because of infectious diseases. Infectious disease pose a major challenge in the elderly for two reasons: The susceptibility of infection increases with age and when infections occur they often present atypically - on the other hand diagnostic uncertainty is much more pronounced in the geriatric population. In addition, geriatric facilities are reservoirs for multi drug-resistant organisms and other nosocomial pathogens and infectious disease consultants also play a vital role in assuring appropriate infection control measures. The prevention, diagnosis and management of infections in geriatric patients, requires the expertise of infectious disease consultants with an interest in this area of medicine. Infectious disease consultants also have a major role in promoting the judicious use of antibiotics in this population because of particularly vulnerable to the inappropriate antibiotic use. While aging is a normal process, and not itself a disease, age related physiological changes place the elderly at high risk for infectious diseases. Susceptibility to infectious diseases in the elderly is increased by combination of factors, including immune senescence, as well as altered skin and mucosal barrier function, degenerative changes in bone and cartilage and reduction in respiratory capacity. Symptoms and signs of

\footnotetext{
* Corresponding author. Al shifa college of Pharmacy, Poonthavanam P.0, Perinthalmanna, Kerala, 67932, India.

E-mail address: dillu7@gmail.com (D. Chandrasekhar).
} 
infections in the elderly may not include the typical features such as fever and chills that clinicians usually rely on to make a clinical diagnosis of infections. This is because of the altered physiological response to infecting pathogen in this patient group, and age -related changes in temperature regulations.

By aging the altered pharmacokinetic changes which plays a wide role in the alteration of antibiotic therapy effectiveness. Polypharmacy has a direct correlation with concomitant disease states. Adverse drug reactions occur more frequently among older patients due to polypharmacy, co morbidities and difficulty with adherence to the therapy as well as age related changes in pharmacokinetics and pharmacodynamics, contributes significantly to the higher incidence. ${ }^{2}$ Many factors such as economics, politics, doctor's knowledge and experience, diagnostic uncertainty, and pharmaceutical marketing led to irrational use of medicines, which can harm patients in terms of poor patient outcome, unnecessary adverse reactions and wastage of resources, often out of pocket payment by patients. Thus emergence of antibiotic resistance is a result of the use, overuse and misuse of antibiotics causing significant mortality and morbidity and extra health care cost. Educational interventions directed at patients and clinicians can increase patient's knowledge and awareness and can also reduce the frequency of inappropriate antibiotic prescription by clinicians. ${ }^{1}$

\section{Methodology}

A prospective interventional study with 12 months duration, were the targets was identified during ward round participation with the doctors and by regular checking of medical records of Gastroenterology, General medicine and Pulmonology departments. The study was conducted and ethically approved by tertiary care referral Hospital, Kerala, Southern India and was not registered under Clinical Trials Registry- India. The full details of the cases including patient name, sex, age, laboratory investigations and antibiotics used for the infections were brought in to the self-designed patient data collection form. After attaining the complete data, the mean age, number of days, frequently used antibiotics, frequent diagnosis, type of antibiotic therapy and trend of antimicrobial prescription pattern with reference to standard guidelines using SPSS 21 version (Statistical Package for Social Science)were analyzed. Also chi square tests were performed to compare the results. The study was carried out into 3 phases, were in the first phase, an observational audit was conducted for all geriatric patients who were admitted more than 2 days were enrolled and monitored from the date of admission until the last day of antibiotic therapy and patients admitted after surgery and who left against medical advices were excluded from the study. Criteria for evaluation includes appropriateness of drug of choice for indication, dose, route of drug administration, and duration of the therapy are assessed. The National guidelines for antimicrobial use version 1.0(2016) was set as the standard for the measuring the appropriateness. The appropriateness of antibiotics usage was assessed by analyzing whether the antibiotic prescribed is justified with National Guidelines for Antimicrobial Use. The second phase was multifaceted intervention aimed to reach all doctors and healthcare professionals. An educational intervention (leaflet) which alerts the irrational use of antibiotics, antibiotic resistance, causes of antibiotic resistance, importance of wise of antibiotics in geriatrics to improve the judicious use of antibiotics by healthcare professionals. Education intervention emphasizing on physicians should be more specific in their diagnosis, as well as the time to time monitoring and reporting of errors in medication administration by nurses. The post intervention audit was conducted in the same manner as in the pre intervention audit, but the audit was restricted to short period due to time constraints and development of adult antibiotic traffic antibiotic light signal (2017) for restriction of antibiotic use[Fig. 5]. The methodology has been illustrated in the below Fig. 1 .

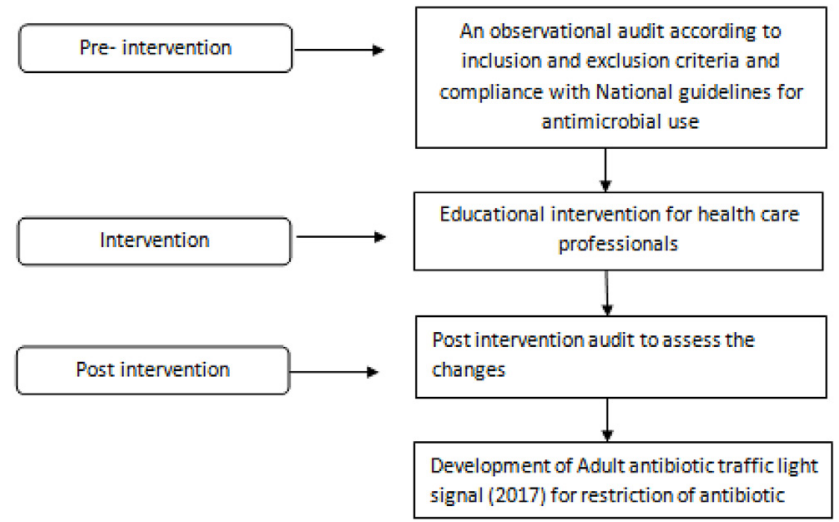

Fig. 1. Illustration of methodology.

\section{Results}

Around 180 patients were included in the study and the prescriptions containing at least one antibiotic were analyzed. There was a male $108(60.0 \%)$ predominance over female $72(40.0 \%)$ and the mean age was found to be $72.23 \pm 6.5$ years. Most of the patients were in the age group of 65-74yrs (63.3\%) and least patients were found to be of $\geq 85$ years $(7.2 \%)$. Out of 180 patients, $63.3 \%(n=114)$ of patients were having $1-2$ co morbidities followed by $36.7 \%(n=66)$ of patients were having greater than three co morbidities. The mean stay duration in pre intervention and post intervention was found to be $7.52 \pm 4.47$ days and $4.93 \pm 1.72$ days respectively. Regarding the duration of hospital stay in the hospital, $42.22 \%(n=76)$ of patients stayed for 5 days, $32.22 \%(n=58)$ of patients stayed for more than 5 days and $25.5 \%$ $(n=46)$ least number of patients stayed for less than 5 days.

Majority of the patients were presented with an acute medical problem on a background of chronic disorders. Alimentary tract and metabolism $(26.02 \%)$ is the most common condition affecting the elderly, followed by infectious diseases and inflammatory (22.80\%) like malaria, pneumonia etc respiratory disorders (20.76\%), cardiovascular disorders (14.16\%), kidney and urinary tract infections (12.28\%) and CNS disorders $(3.50 \%)$. Whereas respiratory tract infection was the most common clinical condition for which antibiotics been prescribed in high percentage (30.6\%) followed by urinary tract infection $(23.9 \%)$, gastrointestinal infections(18.3\%) and fever(16.7\%).

Most of the therapy was empiric in pre intervention (44.44\%) and post intervention $(45.6 \%)$. There was slight increase in the definite therapy in the post intervention $16.6 \%(n=15)$ when compared to pre intervention $13.4 \%(n=12)$. There was no significant $(P<0.753)$ changes in the type of therapy.90\% $(n=162)$ of the patients admitted to the hospital were treated with injections form of antibiotics and only $10 \%(n=18)$ of patients were treated with oral antibiotics (Fig. 2).

In the pre intervention phase, out of 90 cases, $70 \%(n=63)$ was found to be inappropriate which reduced to $48.8 \%(n=44)$ in the post intervention phase. Whereas the percentage of appropriateness got increased to $51.2 \%(n=46)$ from $30 \%(n=27)$ in the pre intervention. $P$ value $=0.004(\mathrm{p}<0.05)$, thus the inappropriateness in the study has statistical significance for the evaluation of appropriateness in geriatric patients.

Out of 90 cases, 63 cases were found to be inappropriate and in the post intervention it get reduced to 44 cases. Most of the inappropriateness was seen in Pulmonology II 19 (30.15\%) which get reduced $11(25 \%)$ in the post intervention followed by Pulmonology I $16(25.39 \%)$ in the pre and reduced to $10(22.72 \%)$. In general medicine I, there was $13(20.63 \%)$ inappropriateness in the pre intervention period which slightly reduced to $10(22.72 \%)$. The least inappropriateness was seen in General medicine II 6(9.52\%) and gastroenterology 9 $(14.28 \%)$ in the pre intervention period and there was a slight 


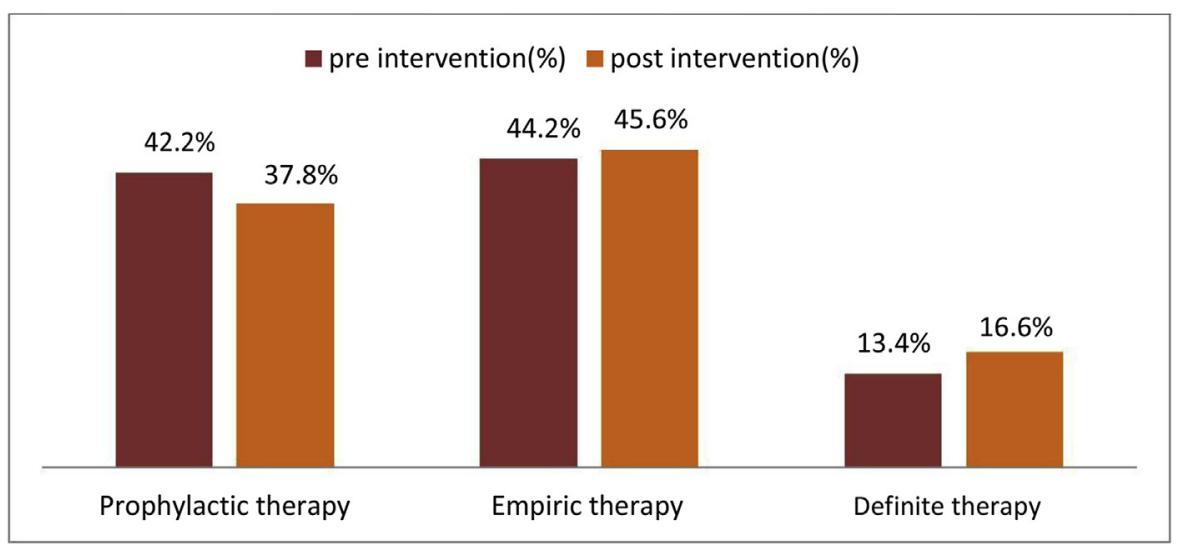

Fig. 2. Type of Therapy.

Table 1

Dept wise inappropriateness.

\begin{tabular}{llcl}
\hline Departments & \multicolumn{2}{l}{ Pre intervention $(\mathrm{n}=63)$} & Post intervention( $\mathrm{n}=44)$ \\
\hline General medicine I & 13 & $(20.63 \%)$ & $10(22.72 \%)$ \\
General medicine II & 6 & $(9.52 \%)$ & $5(11.36 \%)$ \\
Pulmonology I & 16 & $(25.39 \%)$ & $10(22.72 \%)$ \\
Pulmonology II & 19 & $(30.15 \%)$ & $11(25 \%)$ \\
Gastroeneterology & 9 & $(14.28 \%)$ & $8(18.18 \%)$ \\
\hline
\end{tabular}

reduction in the inappropriateness $5(11.36 \%)$ and $8(18.18 \%)$ respectively (Table 1).

Among the 4 indicators, wrong drug duration was the most common inappropriate indicator $(41.2 \%)$ which reduced to $27.8 \%$ in the post intervention phase (Table 2). The second most common reason was wrong drug selection $23.4 \%$ which significantly reduced to $20 \%$. The least common inappropriate indicator was found to be wrong drug route $(1.2 \%)$. All the 4 indicators was found to be reduced in the post intervention phase and this variation was statistically significant $(\mathrm{p}=0.23)$. The study shows that in the pre intervention phase, 27(15\%) were compliant and 63(35\%) were not compliant with the national guidelines (Fig. 3). Where as in the post intervention phase, there was an increase in the compliance $46(25.6 \%)$ with the national guidelines and decrease in the noncompliance 44(24.4\%). Out of 180 patients, 73 patient cases were found to be inappropriate in the pre interventions which get reduced to 54 cases. In this Inappropriate prophylaxis $27.8 \%(n=25)$ was found to be the main reason for inappropriateness in the pre intervention phase which get reduced to $18.8 \%(n=17)$. Use of antibiotics without evidence of infection $20 \%$ ( $\mathrm{n}=35 \%$ ) was another reason in the pre intervention which reduced to $18.8 \%(n=17)$, followed by bacterial resistance $10 \%(n=5)$ which was reduced to $7.8 \%(n=7)$. The reason for inappropriateness $(\mathrm{p}=0.23)$ shows a statistically significant reduction. Whereas, there was a significant increase in the appropriateness $(\mathrm{p}=0.23)$ when pre $(30 \%)$ and post $(51.2 \%)$ was compared (Table 3). Apart from the Gyssens et al. criteria appropriate antibiotic use was evaluated using 4 quality indicators(QIs) - perform culture before starting treatment, prescribe empirical treatment in accordance with national guideline,

Table 2

Gyssens et al. indicators for assessment of inappropriate use of antibiotics.

\begin{tabular}{lll}
\hline Gyssens criteria & Pre intervention & Post intervention \\
\hline 1.Wrong drug selection & $21(23.4 \%)$ & $18(20 \%)$ \\
2.Wrong drug duration & $37(41.2 \%)$ & $25(27.8 \%)$ \\
3.Wrong drug dose & $4(4.4 \%)$ & $1(1.2 \%)$ \\
4.Wrong drug route & $1(1.2 \%)$ & $0(0 \%)$ \\
Appropriateness & $27(30 \%)$ & $46(51.2 \%)$ \\
\hline
\end{tabular}

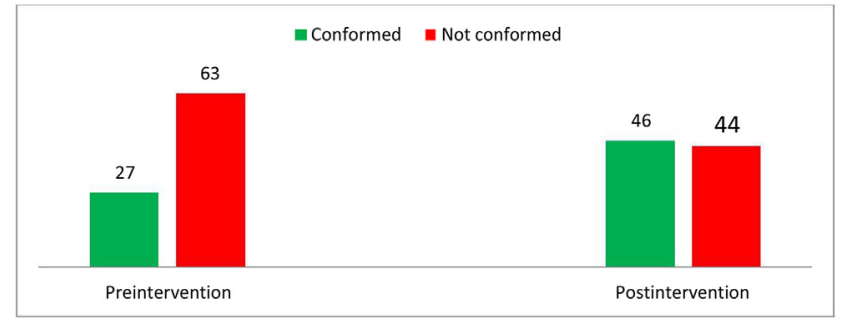

Fig. 3. Compliance of physicians with national guidelines.

Table 3

Reasons for inappropriateness.

\begin{tabular}{lll}
\hline Reasons for inappropriateness & Pre intervention & Post intervention \\
\hline Inappropriate prophylaxis & $27.8 \%$ & $18.8 \%$ \\
Use of antibiotics without any evidence of & $20 \%$ & $18.8 \%$ \\
$\quad$ infection & $10 \%$ & $7.8 \%$ \\
Bacterial resistance & $12.2 \%$ & $3.4 \%$ \\
Narrow spectrum was available & $30 \%$ & $51.2 \%$ \\
Appropriateness & & \\
\hline
\end{tabular}

switch from IV to oral at the time of discharge and de escalate antibiotic treatment on the basis of culture test. Each QIs are monitored for 180 patients, in $30.55 \%(n=55)$ of patients culture before starting treatment was done and in $47.77 \%(n=86)$ the prescription of empirical treatment was in accordance with national guideline. Switching from IV to oral at the time of discharge (93.3\%) was done in most of the patients. De escalation of antibiotic treatment on the basis of culture test was done only in $45.55 \%$ of patients (Fig. 4). The average number of antibiotics per prescription was found to be 2.06. The Anatomical Therapeutic Chemical Classification was used for the classification of antibiotics. 240 antibiotics were prescribed in 180 patients. The profile of antibiotics prescribed is shown in the Fig. 5. Most of the antibiotics prescribed were cephalosporins and related substances $(34.16 \%)$ followed by carbapenam $(29.16 \%)$, quinolones $(28.33 \%)$, Nitrofurantoin (25.41\%),metronidazole (22.08\%), aminoglycosides (17.91\%), beta lactam antibacterials (14.16\%),tetracyclines $(6.25 \%)$ and other antibiotics (5\%). Cefaperazone sulbactum (48.43\%, Cefglobe S) was the most commonly used combinational antibiotic. Amoxicillin + clavulanic acid (32.03\%) and piperacillin + tazobactum (19.53\%) were the other combinational antibiotics used.

\section{Discussion}

Total 180 patients were included in the study. There showed a male predominance with 108 patients (60\%) and females 71(40\%) which is similar to the studies conducted by Sharma $\mathrm{M}$ et al. ${ }^{9}$ Akram Ahmad 


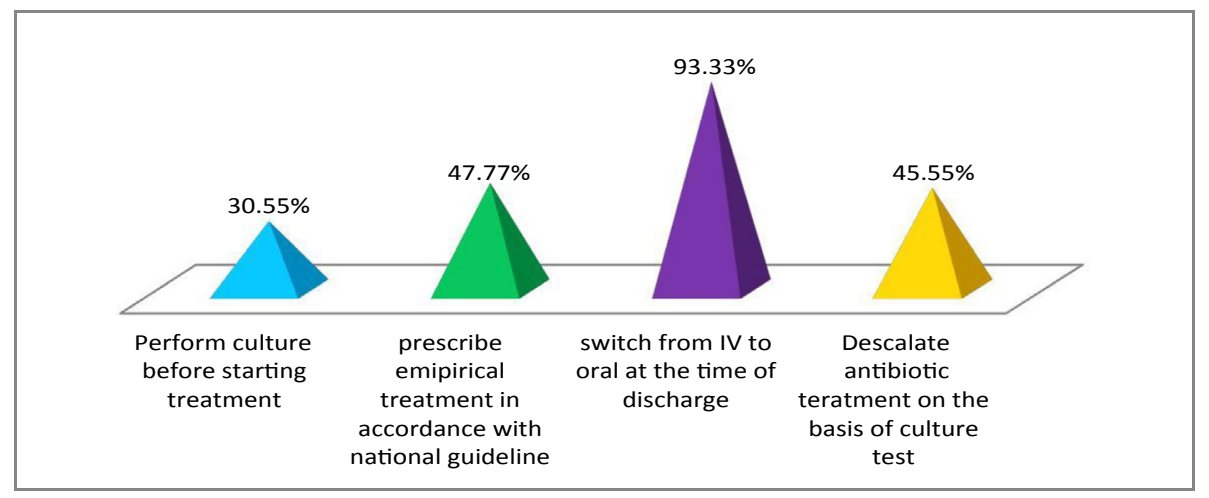

Fig. 4. Quality indicators for Appropriate Antibiotic use.

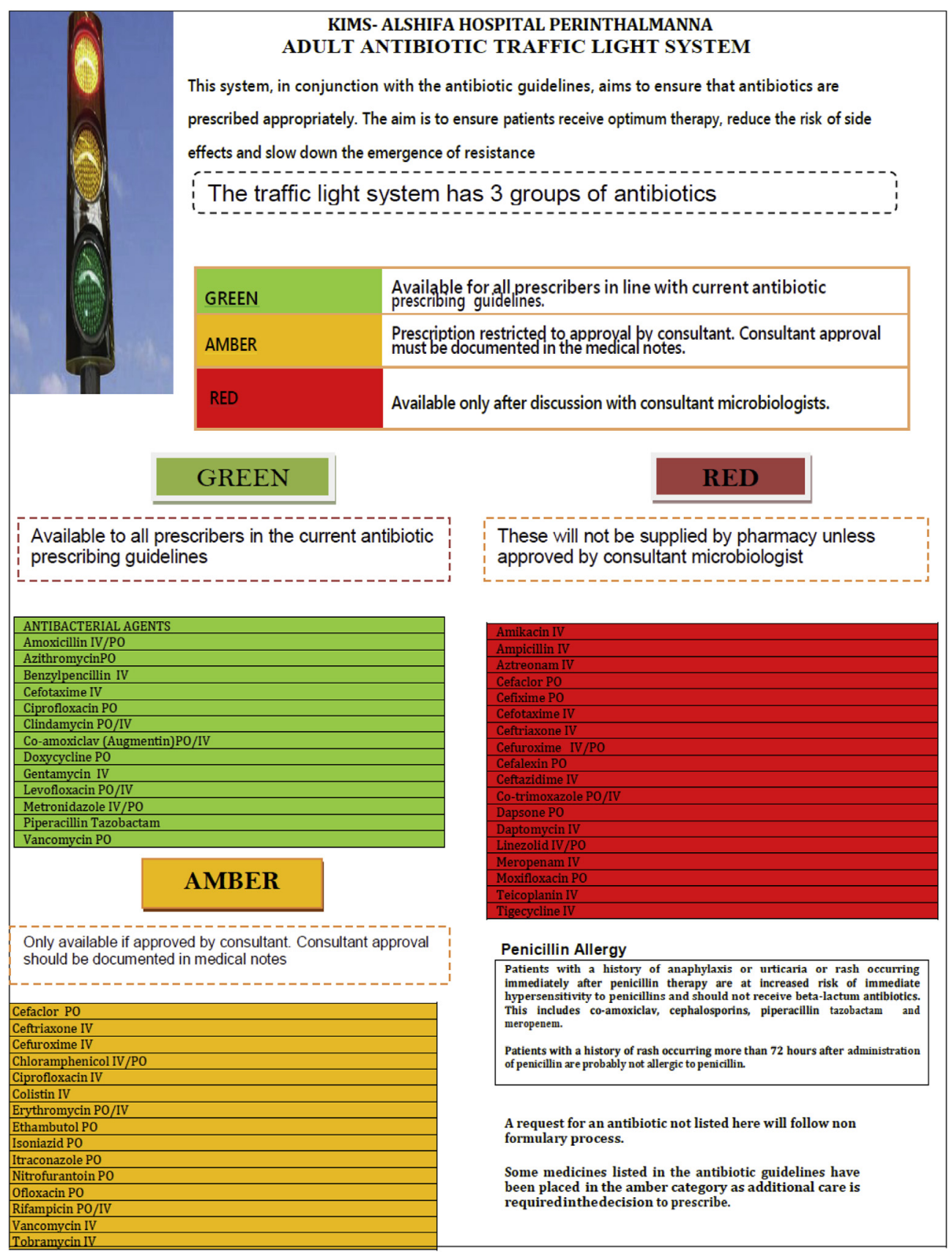

Fig. 5. Adult antibiotic traffic light signal. 
et al. ${ }^{5}$ conducted a study on prescription pattern of antibiotics in the medicine departments of Vydehi institute of medical science and research centre in Bangalore also found that male (55\%) predominance over females (45\%). Akram Ahamad et al. ${ }^{5}$ implies that this may be because the female populations was less exposed to environmental influences when compared to males so they get more infectious diseases. Similar results were found in studies involoving pediatric population conducted by Ramanath et al. ${ }^{6}$ and Akram et al. ${ }^{7}$ Even though the female population is higher in Kerala according to latest census, the male preponderance may have many reasons. One implication is that male patients might be more ill when compared to female patients. Another possibility is for it to be the gender issue, that males receives more medical care for possible reasons such as expectations from patients and his family. But it was opposed by the study conducted by Binit N Jhaveri et al. ${ }^{10}$ which showed a female predominance.

The study identified majority of the population was found to be in the age group of 65-74years. This may be because of the reduction of life expectancy. The study result showed that most of the elderly patients on antibiotic were with 1-2 co morbidities (63.3\%) followed by $>3$ co morbidities (36.7\%). The absence of co-morbidities predicts lesser use of antimicrobial drugs and admission due to non-infectious causes may lead to prolonged stay in hospital and more likelihood of receiving multiple antimicrobial drugs. During the study, an average of $7.52 \pm 4.7$ days hospital stay was observed which reduced to $4.93 \pm 1.72$ days after intervention.

The commonly prescribed medications are a reflection of the pattern of disease burden in the place. Most of the patients were reported to have various associated co-morbid conditions mainly alimentary tract and metabolism disorders (26.02\%), infection and inflammatory diseases $(22.80 \%)$ and respiratory disorders (20.76\%). Whereas in the study conducted by Ankita Bist et $\mathrm{al}^{3}$ at Bidar Institute of Medical sciences in Karnataka were respiratory tract infections (44\%) and GI problems $(25.71 \%)$ were the most common reason for admission.

Respiratory tract infections was the most common condition for which antibiotics were prescribed(30.6\%), followed by Urinary tract infections (23.9\%), GI infections (18.3\%), fever and suspected infections and others (10.6\%). The study findings was also similar to an intervention study in Thailand, conducted by Anucha Apisarnthanarak et al. ${ }^{11}$ were respiratory tract infections in pre and post intervention phase (pre: $45 \%$, post: $48 \%$ ) were the most common conditions leading to antibiotic prescription. The second most common condition was UTI (pre: $12 \%$, post:14\%) followed by gastrointestinal tract infections (pre:6\%,post:5\%), fever and suspected tract infections (pre:5\%; post:3\%) and others (pre:3\%,post:4\%). ${ }^{11}$

The average number of antibiotics per prescription is an important index of the scope for review and educational intervention in prescribing habits. In this study, the average number of antibiotics was found to be high though lower than other findings of others studies. It was found that the average number of antibiotics per prescription was found to be 2.06. The mean number of drugs per prescription should be as low as possible because the higher the number of drugs, the greater the risk of drug resistance, non-compliance as well as cost. On the basis of the result of prescription analysis, most of the population was on treatment with 2 antibiotics (40\%), followed by single antibiotics (31.11\%), 3 antibiotics $(20.55 \%)$ and $8.33 \%$ of patients with $>3$ antibiotics. This result was contrast to the study findings by Bist $A$ et al. $^{3}$ (monotherapy:60\%,2 drugs: $27 \%, 3$ drugs:11.06\%, 4 drugs: $1.40 \%)$.

It has been demonstrated that cephalosporins and related substances (34.16\%), carbapenams (29.16\%), quinolones $(28.33 \%)$ and Nitrofurantoins $(25.41 \%)$ were the most frequently used antibiotics in the hospital. Cephalosporins have continued to be a mainstay of therapy, in hospitals because of their broad spectrum of activity, clinical efficacy and favorable tolerability profiles. Ceftriaxone was the most commonly used antibiotic among monotherapy and Cefaperazone + salbactum (48\%) was the most common combination therapy used. Cephalosporins are generally widely prescribed due to their high potent action, available in various formulations in the market, their extended indications and the activity against gram negative to gram positive bacteria means broad spectrum activity from first generation to third generation of Cephalosporins. Even though aminoglycosides were highly sensitive to commonly isolate organisms of UTIs, they were used less often due to renal toxicity of drugs and age related renal impairment among the patients which is considered to be significant. Amoxicillin clavalunic acid was used because of the broad spectrum of activity and as per the recommendation of CDC.

In the current study, $70 \%$ of inappropriateness was found in the pre intervention phase which reduced to $48.8 \%$ in the post intervention phase, were the appropriateness was increased in the post intervention to $51.2 \%$ from $30 \%$ in the pre intervention. Type of therapy was divided into 3- definitive, empiric and prophylactic. Prescriptions were considered empiric if: (a). the medical records contained information that the antibiotic was presented for therapy (b). an infectious disease was diagnosed(c)clinical signs of infection such as fever were present on the day that antibiotic therapy was initiated. Antibiotics were classified as prophylactic if:(a). the medical record stated that the antibiotic was prescribed for prophylaxis. (b) Antibiotic started before culture test. Antibiotic were considered definite if: (a) antibiotic was given after culture test. The antibiotic therapy was reviewed to assume compliance with recommendations of the national guidelines for antimicrobial use (2016). After intervention there was no significant change yet a slight change in the type of therapy pattern of antibiotics. Antibiotic given without performing culture sensitivity was reduced and definitive therapy was increased. Since geriatric patients were presented with clinical signs of infections the empirical therapy got a slight change.

The gold standards used were National guidelines for antimicrobial use and Gyssens criteria ${ }^{8}$ This study shows that there was compliance of prescriptions with ideal standards such as Gyssens recommendations and the national guidelines for antimicrobial use were less in the pre intervention phase. $70 \%$ has non compliance with the Gyseens recommendation in the pre intervention which reduced to $48.8 \%$ in the post intervention phase. Failure to comply with Gyssens et al. recommendations, may lead to drug resistance, poor patient care outcomes and unnecessary expenditure in an already resource stretched environment. Out of 90 patients, $23.4 \%$ of patients have wrong drug selection in the pre intervention phase which significantly reduced to $20 \%$. This indicated that either the antibiotic given was not specific to the disease or organism. This can be reduced by initiation of antibiotic treatment only after identification of specific organism.

Documentation of antibiotic duration from commencement of therapy to the end of therapy can help to reduced overuse or misuse of antibiotics. This was the main reason found in the study which significantly reduced to $27.8 \%$ from $41.2 \%$. In general, there are still difficulties in documenting duration of treatment or review date and thus there is need to develop policies on prescription requirements. Although this does not reflect good or bad administration of prescribed drugs, it may be interesting to determine for example if antibiotics are administered at correct intervals as stated on the prescriptions. Documentation of dosing frequency is a necessity in all spheres of prescribing drugs. Not indicating the dosing interval is one of the reasons why micro-organisms develop resistance as drugs tend to be administered wrongly especially with concentration dependent antibiotics. Wrong drug dose was not a serious concern since only $4.4 \%$ of inappropriateness was seen in the pre intervention phase which reduced to the $1.2 \%$ in the post intervention phase. The dose should be documented correctly since dose is crucial as it indicates how much drug should be given at each time. The study result indicated that the wrong drug route was not a serious problem in the current circumstances.

Apart from the Gyssens et al. criteria the reason for inappropriateness was also evaluated in pre and post phase of the study, the evaluation parameters are inappropriate prophylaxis(27.8\%), use of 
antibiotics without any evidence of infection(20\%), bacterial resistance $(10 \%)$ and narrow spectrum availability(12.2\%). Through the multifaceted intervention to specifically targeted antibiotic prescribing defects identified during a baseline period of observation has significantly increased the appropriate use of antibiotic. The study result analogues the results of Anucha Apisarnthanarak et al. ${ }^{11}$ were they evaluated the impact of education and an antibiotic program in tertiary hospital. They analyzed the impact on 5 reasons for inappropriateness- Inappropriate surgical prophylaxis, use of antibiotic without any evidence of infection, redundant spectrum, bacterial resistance and narrow spectrum was available.

Antibiotic stewardship programs are increasingly being implemented in hospitals to optimize antibiotic use. Most important in these programs tends to be the provision of guidelines and instructions for prescribers, but this alone will not be sufficient to bring about change and improvement of antibiotic use. Considering the barriers to guidelines on judicious use of an antibiotics during the conception, dissemination and implementation of intervention strategy the four quality indicators are assessed, that were most in need of improvement of quality of antibiotic treatment of hospitalized geriatric inpatients. These QIs enable to determine for which steps along the antibiotic pathway there is room for improvement, and to set priorities for targeted improvement. In this manner, a quality system can be introduced in hospitals to continuously self-monitor and improve the appropriateness of antibiotic use. Culture and sensitivity reports play a vital role in appropriate selection of antibiotics. If a therapy is initiated once after doing a culture and sensitivity it is called definite therapy. The increased definitive therapy can reduce the antibiotic misuse and resistance. In the current study, performance of culture before starting treatment was done only in $30.55 \%$ of patients. The probable reasons for levels of ordering tests were that results took more than five days in which case some patients would even be discharged from hospital before culture results were ready. The other reason can be attributed to ineffective microbiology laboratory. In the current study $47.7 \%$ of patients were prescribed the empirical treatment in accordance with national guidelines. Adherence to such guidelines improves clinical outcome, is correlated with a lower rate of development of resistance to antibiotics, and lowers costs. ${ }^{4}$ The study findings by Caroline $\mathrm{M}$ A van den Bosh et al. ${ }^{4}$ developed quality indicators to measure appropriateness of antibiotics use in the treatment. Inappropriate intravenous therapy increases the cost of care while also exposing the patient to the risk associated with intravenous catheters. Switch therapy, i.e., the change from I.V. to oral treatment, has been studied by several investigators over the past few years, and has been shown to save costs, shorten the length of hospital stays, and decrease the adverse reactions of i. v. administration, all with equal therapeutic outcome. ${ }^{8}$ In our hospital, parenteral administration of antibiotics was more common then oral and most of the parenteral drug was switched to oral form (93.3\%). The National guidelines also did not recognize this point. Generally, in our setting, oral antimicrobial agents are promoted for out-patient general practice and parenteral antimicrobial agents for inpatient hospital practice. Factors such as the unavailability of an oral preparation and a patient's inability to tolerate one may influence the choice of this route. $^{8}$ About $90 \%$ inpatients were on injectable antibiotics and only $10 \%$ were on oral antibiotics. Injectables may be more favored due to fast onset of action. The hospital protocol provides no clear guidelines for the choice of a route which might have been responsible for choosing a parenteral route in the present.

Other important parameter was de-escalation, a mechanism whereby provision of effective initial antibiotic treatment is achieved while avoiding unnecessary antibiotics use that would promote the development of resistance. The embodiment of de-escalation is that based on the microbiology results around the day 3 therapy point; the empiric antibiotics that were started are stopped or reduced in number and/or narrowed in spectrum. ${ }^{12}$ In our settings de-escalation was done only for $45.55 \%$. Thus by this study there was no significant change in the drug prescribing pattern but there was a significant improvement in the quality of antibiotic treatment provided to the patients. Both physicians and pharmacist must be encouraged by national health programs to provide more information about appropriate antibiotic use and its importance while prescribing or dispensing these medications.

\section{Conclusion}

This study investigated the prescribing pattern of antibiotics by physicians, which helps to observe, evaluate, and suggest modifications to improve the prescribing habits of the health care providers. The study found that different physicians follow different guidelines for antimicrobial use that may be reason for poor compliance with national guidelines for antimicrobial use. Updating the guidelines in close collaboration with the specialists involved followed by active dissemination proved to be the efficient way to improve compliance with guidelines recommendations.

Audits of individual patient care were qualitatively performed using Gyssens criteria ${ }^{8}$ and subsequent intervention increased the appropriateness rate. Since the interventions has a positive impact on inappropriateness the hospital should develop a system, structures and processes to monitor and regulate the use of antibiotics in geriatrics to promote the rational use of antibiotics. The adult antibiotic traffic light signal that I had developed based upon the National guidelines for antimicrobial use ver 1.0 (2016) and hospital formulary help to restrict the antibiotic usage. Cephalosporin was the classes of antibiotics were most frequently prescribed and among mostly ceftriaxone was the drug of choice. The choices of antibiotics for different infectious disease differ at different Indian hospitals by physician's choice and preferences. Also, microbiologist, nurses and pharmacists can take part in promoting the rational use of antibiotics, which can lead to their professional betterment. The study findings raise a lot of concern due high level of antibiotics use in geriatrics. It was concluded that giving antibiotics as prophylaxis and without undergoing any blood or other specimen cultures result in antibiotic resistance. Efforts are needed to educate physicians about rational use of antibiotics and also prescribing antibiotics according to microbiological test result can reduce the inappropriate use of antibiotics.

\section{References}

1. Ceyhan M, et al. Inappropriate antimicrobial use in Turkish pediatric hospitals: a multicenter point prevalence survey. PLoS One. 2010;14:e55-e61.

2. Atif Muhammed, et al. Investigation of antimicrobial use at a tertiary care hospital in Southern Punjab. Antimicrob Resist Infect Contr. 2017;6(41):1-12.

3. Bist Ankita, Kulkarni Gajanan P, Gumma KashinathM. Study on prescribing antibiotics in geriatric patients admitted to the medical wards in a tertiary care hospital. Int J Basic Clin Pharmacol. 2016;5(1):155-158.

4. Caroline M, van den Bosch A, Geerlings Suzanne E, Natsch Stephanie, Prins Jan M, Hulscher Marlies EJL. Quality indicators to measure appropriate antibiotic use in hospitalized adults. Clin Infect Dis. 2015;60(2):281-291.

5. Ahmad Akram, et al. Study the prescription pattern of antibiotics in the medicine department in a teaching hospital. Int J Toxicol Pharmacol Res. 2014:6(2):43-46.

6. Ramanath Katta Venkatesh, Balaji BVB. Study the outpatients prescription pattern of antibiotics in paeditric populations of two hospitals. Arch Pharm Pract. 2013;4(1):21-27

7. Ahmad Akram, Parimalakrishnan S, Mohanta Guru Prasad, Patel Isha, Manna PK. A study on utilization pattern of higher generation antibiotics among patients visting community pharamacies in Chindambaram,Tamilnadu at South India. Int J Pharm. 2012;2(3):466-471.

8. Baktygul Kambaralieve, et al. An assessment of antibiotics prescribed at the secondary health care level in the Kyrgyz Republic. Nagoya J Med Sci. 2011;73:157-168.

9. Sharma M, et al. Antibiotic prescribing in two private sector hospitals; one teaching and one non-teaching: a cross-sectional study in Ujjain, India. BMC Infect Dis. 2012;12:155.

10. Jhaveri BN, Patel TK, Barvaliya MJ, Tripathi CB. Drug utilization pattern and pharmacoeconomic analysis in geriatric medical inpatients of a tertiary care hospital of India. J Pharmacol Pharmacother. 2014;5(1):15-20.

11. Apisarnthanarak Anucha, et al. Effectiveness of education and an antibiotic-control program in the tertiary care hospital in Thailand. vol. 42. 2006; 2006:768-775.

12. Masterton Robert G. Antibiotic de -escalation. Crit Care Clin. 2011;27(1):149-162. 